\title{
PIGMENTED VILLONOULAR SYNOVITIS OF THE POPLITEAL FOSSA - CASE REPORT
}

doi: 10.2478/rojost-2018-0025

\author{
M. Pleniceanu ${ }^{1}$, M. Popa ${ }^{1,2}$, M. Nica ${ }^{1,2}$, Z. Panti ${ }^{1,2}$, B. Şerban 1 , R. Ene ${ }^{1,2}$, C. Cîrstoivi, ${ }^{1,2}$ \\ ${ }^{1}$ Orthopedics and Traumatology Department, University Emergency Hospital, Bucharest, Romania \\ 2"Carol Davila" University of Medicine and Pharmacy, Bucharest, Romania
}

Pigmented villonodular synovitis is a benign proliferative condition that causes the synovium of the joints and tendons to thicken and overgrow. The causes have not been completely discovered, with trauma and repetitive inflammation being the most incriminated. Genetic aberrations have been reported in most cases and could suggest a neoplastic origin of the disease. It appears in 2 forms: localized or diffuse. Although it can affect all joints, the most common location is the knee. The treatment options include anti-inflammatory medication, surgical removal (open or arthroscopic) and radiotherapy (mixed results).

We present the case of a 31-year-old male who came to our Clinic with pain and tumefaction of the right popliteal fossa. From the patient's medical history, it resulted that he had developed a tumoral growth in the popliteal fossa 20 months before, which was removed. After extensive investigations (X-ray, CT, and MRI), a biopsy was performed. The result was pigmented villonodular synovitis. The proliferation was removed for the second time. The immediate post-surgery evolution was good and the patient is scheduled for periodic follow-ups.

Although the proliferation was removed entirely, studies show that this disease has a high chance of recurrence ( $46 \%$ for the diffuse form). Also, in time, repeated excisions can force the surgeon to choose more radical options (joint reconstruction or even amputation).

Keywords: synovium, proliferation, surgery, recurrence 